ONLINE MUTATION REPORT

\title{
Common origin of the Val30Met mutation responsible for the amyloidogenic transthyretin type of familial amyloidotic polyneuropathy
}

\author{
H Ohmori, Y Ando, Y Makita, Y Onouchi, T Nakajima, M J M Saraiva, H Terazaki, O Suhr, \\ G Sobue, M Nakamura, M Yamaizumi, M Munar-Ques, I Inoue, M Uchino, A Hata
}

J Med Genet 2004;41:e51 (http://www.jmedgenet.com/cgi/content/full/41/4/e51). doi: 10.1136/jmg.2003.014803

$\mathrm{T}$ he amyloidogenic transthyretin (TTR) type of familial amyloidotic polyneuropathy (FAP [MIM 176300], http:// www.ncbi.nlm.nih.gov/Omim/) is the most common form of hereditary systemic amyloidosis. ${ }^{1-3}$ It is well known that amyloidogenic TTR resulting from gene mutations is a major constituent of amyloid deposits in tissues of patients with FAP. ${ }^{4}$ To date, more than 80 mutations of the TTR gene have been associated with human amyloidosis. ${ }^{5}$ Of those mutations, a mutation changing valine at amino acid 30 to methionine (Val30Met) is the most common, ${ }^{1-3}$ and only patients with this mutation are found in large foci throughout the world. ${ }^{1}$

The clinical manifestations of the TTR Val30Met type of FAP in Japan and Portugal are as follows: (1) the disorder is inherited as an autosomal dominant trait with equal sex distribution and high penetrance; (2) the age at onset is late twenties to early forties; (3) polyneuropathy with sensory dissociation starts in the legs and progresses in an ascending fashion; (4) manifestations of various autonomic dysfunctions such as severe orthostatic hypotension, disturbed bowel movement with constipation and diarrhoea, impotence, and urinary incontinence invariably appear during the course of the disease; and (5) amyloid deposition in loco causes dysfunction of various organs. ${ }^{1-3} 6$ In contrast, the clinical profile of Swedish patients is different: the average age at onset is the middle fifties, and penetrance of the disease is very low. Also, the disease progresses more slowly in Swedish patients than in Japanese and Portuguese patients.

The disorder was first described in Portugal in 1952. ${ }^{8}$ In the 1960s, large foci of patients were found in Japan and Sweden. ${ }^{910}$ In Japan, two large foci of TTR Val30Met type FAP exist: one in Kumamoto prefecture of Kyushu, and the other in Nagano prefecture on the Mainland of Japan. ${ }^{1}$ However, these two foci are geographically distant, and a consanguineous relationship between the foci has not been identified. The issue of whether there is a common origin for a mutant allele in the two foci has not been resolved. Furthermore, Continho ${ }^{11}$ hypothesised that a mutant allele in the Portuguese kindred could be the origin of the mutation for FAP foci throughout the world, including Japan, Europe, North and South America, and Africa. This hypothesis was based only on well-known historical bonds, and thus far it has not been scientifically tested.

Recently, estimation of haplotype in the absence of DNA of related individuals became possible by means of the maximum-likelihood method algorithm. ${ }^{12}$ In addition, the human genome project has continuously provided human genome resources. Almost 200,000 reliable single-nucleotide polymorphisms (SNPs) for the entire genome of the Japanese population are now available (IMS JSNP database, http:// snp.ims.u-tokyo.ac.jp/index.html). Microsatellite information is now also available (GenBank, http://www.ncbi.nlm.

\section{Key points}

- Our work aimed to identify the origin of Val30Met mutation, responsible for the amyloidogenic transthyretin (TTR) type of familial amyloidotic polyneuropathy (FAP) found in Japan and Europe as foci.

- We analysed the TTR gene haplotype of FAP patients from Japan, Portugal, Spain, and Sweden, and of Japanese and Caucasian control subjects with four single nucleotide polymorphisms (SNPs) and five microsatellite markers in the neighbouring gene region, spanning $215 \mathrm{~kb}$ (loci 1-9).

- We observed that the disease-causing haplotype is the same in two major foci in Japan, indicating the existence of a common founder.

- By comparing haplotype among foci, we obtained results indicating that there could be a common founder for Japanese and Portuguese patients, and for Portuguese and Spanish patients, but not for Swedish or other patients.

- These data, plus the history of 16th century trade, lead to the plausible hypothesis of a mutant allele of a Portuguese kindred being the origin of the mutation in most Japanese FAP patients.

nih.gov/Entrez/); assay of this information provides a powerful method for determining the origin of the gene because heterozygosity is usually much higher than that for SNPs. Statistical software can be used to construct a haplotype by using SNPs and microsatellite information together (Arlequin, http://lgb.unige.ch/arlequin/). Thus, we decided to reanalyse the origin of Val30Met mutation with the newly developed methods and resources.

We first collected DNA samples from five foci-two foci in Japan and one each in Portugal, Majorca Island (Spain), and Sweden-and then analysed haplotype structures with four SNPs and five microsatellite markers covering a $215 \mathrm{~kb}$ TTR gene region. We observed major haplotypes close to $50 \%$ in frequency in each focus of the world, which indicated that each focus originated from one founder because the disease is inherited in an autosomal dominant fashion. Furthermore, we obtained support for the hypothesis that the origin of the mutation is common in the Spanish, Portuguese, and Japanese foci but not in the Swedish foci.

Abbreviations: FAP, familial amyloidotic polyneuropathy; SNPs, single nucleotide polymorphisms; TTR, transthyretin 


\section{METHODS}

\section{Study subjects}

A total of 100 DNA samples were collected from FAP patients with TTR Val30Met in four countries. In Japan, in addition to patients in the two major foci ( 16 patients from Kumamoto and two from Nagano), 20 patients from other locations throughout the country agreed to participate in this study. In Europe, 21 samples from Majorca Island (Spain), 18 samples from Portugal, and 23 samples from Sweden were collected. A total of 54 healthy volunteers in Asahikiawa Medical College were recruited to provide DNA samples for haplotype analysis of the Japanese population, and 96 Caucasian DNA samples were purchased from the Coriell Cell Repository (Coriell Institute, Camden, NJ, USA). The Ethical Committee of Kumamoto University School of Medicine approved this study, and all patients and Japanese volunteers gave written informed consent.

\section{Microsatellite genotyping}

We selected five informative microsatellite sequences (L1, L2, L4, L8, and L9) near the TTR gene region from the National Center for Biotechnology Information (NCBI) database GenBank (fig 1). PCR primers were designed to flank the repeat sequences for the analysis. The PCR primers were as follows: Ll forward: 5'-TGCAACGAAGCTTCAAGAGA-3'; L1 reverse: 5'-AAGCAAATTGCAATGGGAAG-3'; L2 forward: 5'-AGGCAGTGGGATAGTCATGG-3'; L2 reverse: 5'-GGATTCC CTGGGCTCATTAT-3; L4 forward: 5'-TTCCTCCTGGCCGAC TTATT-3'; L4 reverse: 5' -TAGTGCTCCAAACCGGACTT-3'; L8 forward: 5'-AACCTGGGAGATAGAGCTTGC-3'; L8 reverse: 5'-TCTCCTTAATCTAAGAAAGCCACAT-3'; L9 forward: 5'GGCCGTCACAGGTCATAGAA-3'; L9 reverse: 5'-CCACCAGA GGAAACCAACCT-3'. Fluorescence-labeled primers were used for genotyping. PCR was performed in a volume of $7.5 \mu \mathrm{l}$ containing $20 \mathrm{ng}$ of genomic DNA, $10 \mathrm{mM}$ Tris- $\mathrm{HCl}$ (pH 8.4), $50 \mathrm{mM} \mathrm{KCl}, 1.5 \mathrm{mM} \mathrm{MgCl}_{2}, 0.01 \%$ gelatin, $200 \mu \mathrm{M}$ dNTPs, each primer at 5 pmol, and 0.25 U AmpliTaq Gold polymerase (Applied Biosystems, Foster City, CA). PCR amplification was performed for 40 cycles at $95^{\circ} \mathrm{C}$ for $30 \mathrm{~s}$, at $53-60^{\circ} \mathrm{C}$ for $30 \mathrm{~s}$, and at $72^{\circ} \mathrm{C}$ for $30 \mathrm{~s}$, depending on the region analysed, with a final extension step of $5 \mathrm{~min}$ at $72^{\circ} \mathrm{C}$ in a Gene Amp PCR9700 System (Applied Biosystems, Foster City, CA). Electrophoresis was performed with an ABI 310 DNA sequencer; the data were extracted by GeneScan Analysis software (Applied Biosystems, Foster City, CA).

\section{SNP genotyping}

Four SNPs-L3, L5, L6, and L7-were identified from the IMS-JST JSNP database (fig 1). PCR was performed in a volume of $12.5 \mu \mathrm{l}$ containing $20 \mathrm{ng}$ of genomic DNA, $10 \mathrm{mM}$ Tris-HCl (pH 8.4), $50 \mathrm{mM} \mathrm{KCl,} 1.5 \mathrm{mM} \mathrm{MgCl}_{2}, 0.01 \%$ gelatin, $200 \mu \mathrm{M}$ dNTPs, each primer at 10 pmol, and $0.25 \mathrm{U}$ AmpliTaq Gold polymerase. PCR amplification was performed for 40 cycles at $94^{\circ} \mathrm{C}$ for $30 \mathrm{~s}$, at $49-62^{\circ} \mathrm{C}$ for $30 \mathrm{~s}$, and at $72{ }^{\circ} \mathrm{C}$ for $30 \mathrm{~s}$, depending on the region analysed, with a final extension step of $5 \mathrm{~min}$ at $72^{\circ} \mathrm{C}$ in a Gene Amp PCR9700 System (Applied Biosystems, Foster City, CA). The sequencing reaction and electrophoresis were performed with the BigDye Terminator kit (Applied Biosystems, Foster City, CA), according to the manufacture's protocol.

\section{Haplotype estimation and statistical analysis}

Haplotype analysis was done by using the Arlequin program, which is software for population genetic data analysis, and SNPAlyze (DYNACOM, Mobara, Chiba, Japan). Maximumlikelihood haplotype frequencies were computed using an expectation-maximisation (EM) algorithm. This procedure is an interactive one aimed at obtaining maximum-likelihood estimates of haplotype frequencies from multilocus genotype data when the gametic phase is unknown. In this case, simple gene counting is not possible because several genotypes are possible for individuals who are heterozygotic at more than one locus. The computation of the EM algorithm involves the following: start with arbitrary estimates of haplotype frequencies; use these estimates to compute expected genotype frequencies for each phenotype, if one assumes a Hardy-Weinberg equilibrium (the E-step); use the relative genotype frequencies as weights for their two constituent haplotypes in a gene-counting procedure, which leads to new estimates of haplotype frequencies (the M-step); repeat steps $\mathrm{E}$ and $\mathrm{M}$ until the haplotype frequencies reach equilibrium. ${ }^{12}$

\section{RESULTS AND DISCUSSION}

Nine polymorphisms were employed in the present study. Of those, five (L1, L2, L4, L8, and L9) are microsatellite markers (fig 1). L2 and L4 were in the $5^{\prime}$-flanking region of the gene, L1 was located about $90 \mathrm{~kb}$ upstream of L2, and L8 was about $103 \mathrm{~kb}$ downstream of L7. Thus, the gene region analysed covered $215 \mathrm{~kb}$ (fig 1). The heterozygosity of microsatellite markers of Japanese and Caucasian controls is shown in table 1 . All but L2, whose name is D18S36, are anonymous in the GenBank database. All markers except Ll showed similar heterozygosities in Japanese and Caucasians subjects. Four SNPs used in this study (L3, L5, L6, and L7) were obtained from the Japanese SNP database (IMS-JST JSNP). Two of those (loci 3 and 5) were located in the 5'-flanking region, and the remaining two (loci 6 and 7) were located in intron 3, as shown in fig 1 and table 2. Almost no ethnic difference in allele frequency between Japanese and Caucasian control subjects was found. The Val30Met mutation occurs in exon 2 (fig 1).

All nine polymorphisms were employed to construct haplotypes for Japanese patients and controls. To clarify the origin of the mutant allele, Japanese patients were classified into three groups: 16 patients from Kumamoto, two from Nagano, and 20 from the rest of Japan. As shown in table 3, patients from Kumamoto showed a major haplotype frequency of $40 \%$ (which is close to $50 \%$ ), but the same haplotype in the controls was very rare, in fact, it was

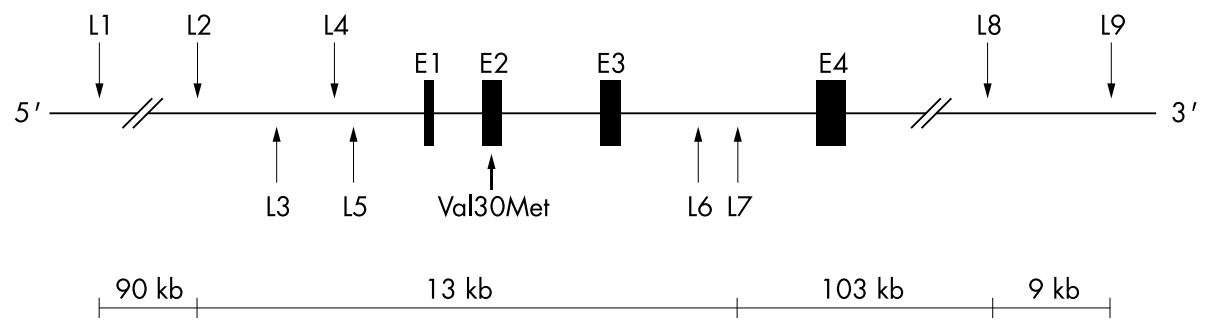

Figure 1 Genomic structure and locations of polymorphic sites used in the human $\Pi R$ gene. Boxes indicate exons; horizontal lines represent introns and the $5^{\prime}$ upstream and $3^{\prime}$ downstream regions of the gene. Downward arrows indicate locations of microsatellite markers, and upward arrows indicate locations of SNPs. The thick upward arrow represents the position of the Val30Met mutation. 
Table 1 Heterozygosity of microsatellite markers above Japanese and Caucasian subjects

\begin{tabular}{lll}
\hline & Japanese & Caucasian \\
\hline L1 & 0.497 & 0.704 \\
L2 & 0.766 & 0.702 \\
L4 & 0.349 & 0.448 \\
L8 & 0.737 & 0.761 \\
L9 & 0.871 & 0.856 \\
\hline
\end{tabular}

undetectable by our method. The most frequent haplotype $(9.6 \%)$ in the controls was different in loci 1 and 9 from the major haplotype of the patients. When a haplotype exists with close to $50 \%$ frequency in patients of one focus of an autosomal dominant disease, the allele could be considered a disease-causing one, and the haplotype, a founder haplotype. Thus, the haplotype, 237-307-T-271-C-G-A-299-311, actually represents the disease-causing allele in this group. Although only two patients from Nagano were available for analysis, which is not sufficient for haplotype construction, it is evident from genotype data that they shared exactly the same haplotype with the Kumamoto patients (table 3). Furthermore, in the other Japanese patients the same haplotype had a frequency of $31.8 \%$, with frequencies of other haplotypes being less than $5 \%$.

To determine whether all major FAP foci throughout the world could be derived from a Portuguese mutant allele, ${ }^{11}$ we analysed the haplotype pattern of patients from Portugal, Spain, and Sweden. We found that all European foci have their own major haplotypes, whose frequencies were close to $50 \%(40.9-47.6 \%)$, which indicated that these haplotypes represent a disease-causing allele, as for the Japanese focus (table 4). When the major haplotype of Portuguese patients was compared with that of Japanese patients, all loci except L8 were exactly the same, which indicated that at least the $103 \mathrm{~kb}$ region (loci $1-7$ ) was common between the two populations. Also, comparison of Portuguese and Spanish patients revealed that at least the $116 \mathrm{~kb}$ region (loci 2-8) was common. However, Swedish patients shared only the $13 \mathrm{~kb}$ region (loci 2-7), which is also a major haplotype in the Caucasian controls, with other foci of patients.

Several major issues remain unsettled in the field of research on FAP caused by the Val30Met mutation of the TTR gene. First, although large foci of patients are located in several restricted areas of the world, why is only the Val30Met mutation found in those foci, even though more than 80 different mutations of TTR gene have been identified? ${ }^{5}$ Second, does any common founder exist among the large foci of patients throughout the world? Third, what causes the significant differences in clinical symptoms among FAP patients with the Val30Met mutation compared with FAP patients with other mutations? For example, the age at onset of the disease in patients in Sweden is much later than that in other countries such as Japan and Portugal. ${ }^{3} 13$
To answer the second question, haplotype analysis via gene polymorphisms has been performed to clarify the founder effect. Several researchers have discussed the origin of the TTR Val30Met gene in FAP patients from Japan, ${ }^{14}$ North America, ${ }^{15}$ and Europe. ${ }^{16}{ }^{17}$ All these data were analysed by using six or seven SNPs inside the gene, a region of about $7.0 \mathrm{~kb}$, and the haplotype was determined by pedigree analysis. Yoshioka et al ${ }^{14}$ analysed eight families, including two from each major focus in Japan. They concluded that the mutation had a multiple origin because two isolated families, not from the two large foci, have different haplotypes, although the remaining six have the same haplotype, which was named "haplotype I". Ii and Sommer ${ }^{15}$ analysed six unrelated FAP patients in North America and performed haplotype analysis in the absence of DNA samples for relatives. They found four different haplotypes and thus agreed with a multiple origin hypothesis. For Europe, however, Almeida et $a l^{16}$ and Reilly et $a l^{17}$ found only haplotype I in patients from Portugal, Spain, and Sweden. In the present study, we used, in addition to four SNPs (two the same as those analysed before by researchers), four highly polymorphic microsatellite markers covering $215 \mathrm{~kb}$ of the TTR gene region. A microsatellite repeat is generally considered to have a relatively high mutation rate when compared with SNPs, but its mutation rate is estimated to be about $10^{-3}$ per locus per generation, ${ }^{18}$ which is negligibly low. If the duration of one generation is assumed to be 30 years, a mutation at a certain microsatellite locus would occur at a rate of only one in 30,000 years. In view of the high heterozygosity of microsatellite markers, such a microsatellite would be a powerful marker for our study.

To determine the haplotype profile in the general population, 54 Japanese and 96 Caucasian control subjects underwent genotyping, and haplotypes were constructed. As expected, haplotypes constructed with all nine polymorphisms had a low frequency, the highest frequencies being $9.6 \%$ in Japanese and $9.5 \%$ in Caucasians, which indicated recombination in this $215 \mathrm{~kb}$ region, which occurred over a substantial time period, as is usual (table 4).

In diseases inherited in an autosomal dominant fashion, an allele responsible for the disease in the patient population would theoretically have a frequency of $50 \%$. The fact that the frequency of the major haplotype observed in the Kumamoto focus (237-307-T-271-C-G-A-299-311) was 40\%, which is considered to be close to $50 \%$, whereas that of Japanese controls was extremely low, indicates that the haplotype represents the disease-causing allele. Although only two patient samples were available from Nagano, the location of another major focus of the disease in Japan, genotype data revealed that they had exactly the same haplotype as that of the Kumamoto patients (table 3). These data strongly support the existence of a relatively recent common founder. As for other FAP patients from the rest of Japan, ${ }^{19}$ a majority of these cases were thought to result from an independent mutational event; ${ }^{20}$ however, an unexpectedly high frequency, $31.8 \%$, of the same haplotype was found, which

Table 2 Positions and allele frequencies of SNPs

\begin{tabular}{lllll}
\hline & & & \multicolumn{2}{l}{ Allele frequency } \\
\cline { 4 - 5 } SNP & ID & Position & Japanese & Caucasian \\
\hline L3 & IMS-JST 118368 & $-2015 T>C$ & 0.712 & 0.665 \\
L5 & IMS-JST 118365 & -1131 C $>$ T & 0.981 & 0.964 \\
L6 & IMS-JST 118364 & IVS3+1242C $>$ A & 0.750 & 0.660 \\
L7 & IMS-JST 152576 & IVS3+1753T>G & 0.713 & 0.670 \\
\hline
\end{tabular}


Table 3 Major haplotype frequencies for Japanese patients and controls

\begin{tabular}{|c|c|c|c|c|c|c|c|c|c|c|}
\hline Population & LI & L2 & L3 & L4 & L5 & L6 & L7 & L8 & L9 & Frequency \\
\hline Kumamoto & 237 & 307 & $\mathrm{~T}$ & 271 & C & G & $A$ & 299 & 311 & 0.400 \\
\hline \multirow[t]{2}{*}{ patients } & 235 & 307 & $T$ & 271 & C & G & A & 299 & 327 & 0.050 \\
\hline & 235 & 311 & $\mathrm{~T}$ & 271 & C & G & A & 305 & 311 & 0.050 \\
\hline \multirow[t]{2}{*}{ Nagano patients } & $237 / 237$ & $305 / 307$ & $\mathrm{~T} / \mathrm{T}$ & $271 / 271$ & $\mathrm{C} / \mathrm{C}$ & $G / G$ & $\mathrm{~A} / \mathrm{A}$ & $299 / 301$ & $311 / 315$ & \\
\hline & $237 / 237$ & $305 / 307$ & $\mathrm{~T} / \mathrm{T}$ & $271 / 271$ & $\mathrm{C} / \mathrm{C}$ & G/G & $\mathrm{A} / \mathrm{A}$ & $299 / 301$ & $311 / 315$ & \\
\hline Patients from & 237 & 307 & $\mathrm{~T}$ & 271 & C & G & A & 299 & 311 & 0.318 \\
\hline \multirow[t]{4}{*}{ other locations } & 237 & 307 & $\mathrm{~T}$ & 271 & $\mathrm{C}$ & G & $A$ & 291 & 333 & 0.045 \\
\hline & 237 & 307 & $T$ & 271 & C & $G$ & A & 299 & 317 & 0.045 \\
\hline & 237 & 307 & $\mathrm{~T}$ & 271 & C & $G$ & C & 299 & 311 & 0.045 \\
\hline & 237 & 305 & $T$ & 271 & C & $G$ & A & 301 & 315 & 0.045 \\
\hline Japanese & 241 & 307 & $\mathrm{~T}$ & 271 & C & $G$ & $A$ & 299 & 325 & 0.096 \\
\hline \multirow[t]{9}{*}{ controls } & 241 & 307 & $T$ & 271 & C & G & A & 299 & 329 & 0.075 \\
\hline & 241 & 307 & C & 269 & C & G & C & 299 & 311 & 0.038 \\
\hline & 241 & 307 & $\mathrm{~T}$ & 271 & C & G & A & 303 & 311 & 0.038 \\
\hline & 241 & 305 & $\mathrm{~T}$ & 271 & $C$ & G & $A$ & 299 & 327 & 0.029 \\
\hline & 241 & 307 & $T$ & 271 & C & G & A & 305 & 311 & 0.029 \\
\hline & 241 & 309 & $\mathrm{~T}$ & 271 & C & G & A & 307 & 311 & 0.019 \\
\hline & 241 & 313 & C & 269 & C & $T$ & A & 299 & 329 & 0.019 \\
\hline & 241 & 307 & $\mathrm{~T}$ & 271 & C & $G$ & $C$ & 299 & 317 & 0.011 \\
\hline & 241 & 307 & $T$ & 271 & C & G & A & 301 & 329 & 0.011 \\
\hline
\end{tabular}

suggests that most were also derived from the same founder of the foci, rather than from independent sporadic mutations.

Each focus of European FAP patients has its own major haplotype, with a frequency close to $50 \% \quad(40.9-47.6 \%)$ (table 4). The major haplotypes for Portuguese and Japanese patients are the same except for locus 8; in other words, at least the $103 \mathrm{~kb}$ region from locus 1 to locus 7 is common. One plausible explanation for this would be that the Portuguese Val30Met mutation was brought to Japan in recent times. Portuguese merchants arrived in Japan during the 16th century, and active trading was pursued in all areas in Kyushu (the island on which Kumamoto is located) until the beginning of the 17th century, when Japan became a closed country. ${ }^{11}$ The most likely explanation is thus as follows: in the early phase of transfer of the allele into the Japanese population, recombination with the Japanese allele (L8-L9; 299-311, which was found in Japanese controls) occurred once between L7 and L8, after which the allele with the Val30Met mutation spread to Nagano and other areas of Japan.

Comparison of the major haplotypes for Portuguese and Spanish patients showed that a $116 \mathrm{~kb}$ region (loci 2-8) was common. This could also be explained by the existence of a common founder. Swedish patients, however, had only a $13 \mathrm{~kb}$ region (loci 3-7) in common with patients of other foci. Although the possibility of a common founder of Swedish and Portuguese cases cannot be ruled out, the fact that the haplotype of this $13 \mathrm{~kb}$ region is the same as the major haplotype found in Caucasian controls might indicate that the Val30Met mutation of the Swedish focus is due to an independent mutational event. However, further molecular genetic studies have to be performed focusing on the Swedish cases because of a trade history including the Swedish Viking invasion of Portugal in the 10th century, and because far milder symptoms occur in Swedish patients than in Japanese or Portuguese patients. The cause of the discrepant clinical phenotypes of Swedish patients and patients from other foci-the third question mentioned above-could be a difference in genetic background such as the existence of modifying gene(s) and/or other known or unknown mechanisms. The answer to this question would be quite valuable for development of therapeutic measures for FAP patients.

Because the haplotypes of Spain and Portugal are somewhat diverse and that of Japan has a single disease-causing allele, the European haplotype could be older than the Japanese haplotype. This suggestion would support the hypothesis that the Portuguese mutant allele was brought to Japan, rather than transferred in an opposite direction, from Japan to Portugal.

In summary, our data provide the molecular evidence that the Val30Met mutation that is widespread among patients in Portugal, Spain, and Japan, results from an ancient mutation of common origin rather than from multiple recurrent mutational events in a common haplotype. The data also

Table 4 Major haplotype frequencies for foci of FAP patients and for controls

\begin{tabular}{|c|c|c|c|c|c|c|c|c|c|c|}
\hline Population & LI & L2 & L3 & L4 & L5 & L6 & L7 & L8 & L9 & Frequency \\
\hline \multirow[t]{3}{*}{ Kumamoto patients } & 237 & 307 & $T$ & 271 & C & $G$ & A & 299 & 311 & 0.400 \\
\hline & 235 & 307 & $\mathrm{~T}$ & 271 & C & G & A & 299 & 327 & 0.050 \\
\hline & 235 & 311 & $\mathrm{~T}$ & 271 & C & G & A & 305 & 311 & 0.050 \\
\hline \multirow[t]{4}{*}{ Japanese controls } & 241 & 307 & $\mathrm{~T}$ & 271 & C & G & $A$ & 299 & 325 & 0.096 \\
\hline & 241 & 307 & $\mathrm{~T}$ & 271 & $\mathrm{C}$ & $G$ & A & 299 & 329 & 0.075 \\
\hline & 241 & 307 & C & 269 & C & G & C & 299 & 311 & 0.038 \\
\hline & 241 & 307 & $\mathrm{~T}$ & 271 & C & G & A & 303 & 311 & 0.038 \\
\hline Portugese patients & 237 & 307 & $\mathrm{~T}$ & 271 & C & G & $A$ & 291 & 311 & 0.438 \\
\hline Spanish patients & 235 & 307 & $\mathrm{~T}$ & 271 & C & G & A & 291 & 321 & 0.476 \\
\hline \multirow[t]{2}{*}{ Swedish patients } & 241 & 307 & $\mathrm{~T}$ & 271 & C & G & $A$ & 303 & 329 & 0.409 \\
\hline & 237 & 307 & $\mathrm{~T}$ & 271 & $\mathrm{C}$ & $G$ & A & 291 & 311 & 0.087 \\
\hline \multirow[t]{4}{*}{ Caucasian controls } & 241 & 307 & $\mathrm{~T}$ & 271 & C & G & $A$ & 299 & 329 & 0.095 \\
\hline & 241 & 307 & $\mathrm{~T}$ & 271 & C & G & A & 299 & 325 & 0.026 \\
\hline & 241 & 307 & $\mathrm{~T}$ & 271 & C & G & $A$ & 299 & 331 & 0.026 \\
\hline & 241 & 313 & C & 269 & $\mathrm{C}$ & $\mathrm{T}$ & C & 303 & 319 & 0.026 \\
\hline
\end{tabular}


clearly demonstrate that microsatellite analysis must be applied in combination with SNPs when the origin and distribution of mutations are studied.

\section{ACKNOWLEDGMENTS}

We wish to thank the patients and their families for their invaluable participation and cooperation. We are grateful to Shin-ichi Chiba and Makiko Funamizu for excellent technical assistance.

\section{Authors' affiliations}

H Ohmori, M Uchino, Department of Neurology, Kumamoto University School of Medicine, Kumamoto, Japan

Y Ando, M Nakamura, Department of Laboratory Medicine, Kumamoto University School of Medicine, Kumamoto, Japan

Y Makita, Department of Pediatrics, Asahikawa Medical College, Asahikawa, Japan

Y Onouchi, A Hata, Laboratory for Gastrointestinal Diseases, RIKEN SNP Research Center, Yokohama, Japan

T Nakajima, I Inove, Division of Genetic Diagnosis, The Institute of Medical Science, The University of Tokyo, Japan

M J M Saraiva, H Terazaki, Instituto de Ciencias Biomedicas, Universidade de Porto, Porto, Portugal

O Suhr, Department of Medicine, Umea University Hospital, Umea, Sweden

G Sobue, Department of Neurology, Nagoya University Graduate School of Medicine, Nagoya, Japan

M Yamaizumi, Institute of Molecular Embryology and Genetics, Kumamoto University, Kumamoto, Japan

M Munar-Ques, Hospital General Universitario, Murcia, Spain

A Hata, Department of Public Health, Chiba University Graduate School of Medicine, Chiba, Japan

The authors' work was supported by grants from the Amyloidosis Research Committee, the Pathogenesis, Therapy of Hereditary Neuropathy Research Committee, the Surveys and Research on Specific Disease, the Ministry of Health and Welfare of Japan, and the Charitable Trust Clinical Pathology Research Foundation of Japan, and Research for the Future Program Grant and Grant-in-Aid for Scientific Research on Priority Areas (C) "Medical Genome Science", and Grantsin-Aid for Scientific Research (B) 15390275 from the Ministry of Education, Science, Sports and Culture of Japan.

Conflict of interest: none declared.

Correspondence to: Dr A Hata, Department of Public Health, Chiba University Graduate School of Medicine, Inohana 1-8-1, Chiba 2608670, Japan; ahata@med.m.chiba-u.ac.jp

Received 29 October 2004

Accepted 2 November 2004

\section{REFERENCES}

1 Araki S. Type I familial amyloidotic polyneuropathy (Japanese type). Brain Dev 1984;6:128-33

2 Benson MD. Familial amyloidotic polyneuropathy. Trends Neurosci 1989;12:88-92.

3 Ando Y, Araki S, Ando M. Transthyretin and familial amyloidotic polyneuropathy. Intern Med 1993;32:920-22.

4 Tawara S, Nakazato M, Kangawa K, Matsuo H, Araki S. Identification of amyloid prealbumin variant in familial amyloidotic polyneuropathy (Japanese type). Biochem Biophys Res Commun 1983;116:880-8.

5 Connors LH, Richardson AM, Theberge R, Costello CE. Tabulation of transthyretin (TTR) variants as of 1/1/2000. Amyloid 2000;7:54-69.

6 Ando Y, Suhr OB. Autonomic dysfunction in familial amyloidotic polyneuropathy (FAP). Amyloid 1998;5:288-300.

7 Drugges U, Andersson R, Chizari F, Danielsson M, Holmgren G, Sandgren O, Sousa A. Familial amyloidotic polyneuropathy in Sweden: a pedigree analysis. J Med Genet 1993;30:388-92.

8 Andrade C. A peculiar form of peripheral neuropathy. Familial atypical generalized amyloidosis with special involvement of the peripheral nerves. Brain 1952;75:408-27.

9 Andersson R, Kassman T. Vitreous opacities in primary familial amyloidosis. Acta Ophthalmol 1968;46:441-7.

10 Araki S, Mawatari S, Ohta M, Nakajima A, Kuroiwa Y. Polyneuritic amyloidosis in a Japanese family. Arch Neurol 1968;18:593-602.

11 Continho P. Travels of a gene: a tale of the migrations of familial amyloidotic polyneuropathy (Portuguese type). Porto, Portugal: ROCHA/Artes Graficas, 1989.

12 Excoffier L, Slatkin M. Maximum-likelihood estimation of molecular haplotype frequencies in a diploid population. Mol Biol Evol 1995;12:921-7.

13 Continho P, Desilva AM, Lima JL, Barbosa AR. Forty years of experience with type I amyloid neuropathy: review of 483 cases. In: Glenner GG, Costa PP, de Freitas AF, eds. Amyloid and amyloidosis. Amsterdam: Excerpta Medica, 1980:88-98.

14 Yoshioka K, Furuya H, Sasaki H, Saraiva MJ, Costa PP, Sakaki Y. Haplotype analysis of familial amyloidotic polyneuropathy. Evidence for multiple origins of the Val-Met mutation most common to the disease. Hum Genet 1989;82:9-13.

15 li S, Sommer SS. The high frequency of TRR $M^{30}$ in familial amyloidotic polyneuropathy is not due to a founder effect. Hum Mol Genet 1993:2:1303-5.

16 Almeida MR, Aoyama-Oishi N, Sakaki Y, Holmgren G, Ulf D, Ferlini A Salvi F, Munar-Oues M, Benson MD, Skinner M, Costa PP, Saraiva MJ. Haplotype analysis of common transthyretin mutations. Hum Genet 1995; $96: 350-4$

17 Reilly MM, Adams D, Davis MB, Said G, Harding AE. Haplotype analysis of French, British and other European patients with familial amyloid polyneuropathy (met 30 and tyr 77). J Neurol 1995;242:664-8.

18 Weber JL, Wong C. Mutation of short tandem repeats. Hum Mol Genet 1993;2:1123-8.

19 Misu K, Hattori N, Nagamatsu M, Ikeda S, Ando Y, Nakazato M, Takei Y, Nanyu N, Usui Y, Tanaka F, Harada T, Inukai A, Hashizume Y, Sobue G. Late-onset familial amyloid polyneuropathy type I (transthyretin Met30associated familial amyloidotic polyneuropathy) unrelated to endemic focus in Japan: clinicopathological and genetic features. Brain 1999;122:1951-62.

20 Ikeda S, Nakazato M, Ando Y, Sobue G. Familial transthyretin-type amyloid polyneuropathy in Japan: clinical and genetic heterogeneity. Neurology 2002;58:1001-7. 